\title{
Das vietnamesische Lehrstück des kleinbürgerlichen Anti-Imperialismus ${ }^{\dagger}$ )
}

\section{Ruideger Baron}

Nachdem durch das Waffenstillstandsabkommen vom 27. Januar 1973 jetzt der grobe Rahmen für den Ausgang des III. Indochinakrieges gesetzt zu sein scheint, wird es endgültig Zeit für die in den Jahren des Vietnamkrieges entstandene Neue Linke in den kapitalistischen Metropolen, ihr idealistisches Verhältnis zu dem Befreiungskampf der Völker Indochinas zu überprüfen; es muß endlich möglich sein, auch die Politik der Kommunisten in Indochina der Kritik zu unterwerfen, was insbesondere deshalb geboten zu sein scheint, weil inzwischen unübersehbar geworden ist, was der Vietnam-Protest nie wahr haben wollte und wogegen sich die Sektierer aller Schattierungen bis heute noch mit der Phrase vom großen Sieg des vietnamesischen Volkes hinwegzutäuschen versuchen: Auch nach dem jetzt erfolgten fast vollständigen Abzug der Amerikaner aus Vietnam bestehen kaum Aussichten für eine Machtübernahme der Befreiungsfront!

I

Was die USA seit den frühen Fünfzigerjahren für Indochina anstreben, scheint jetzt in Vietnam auf absehbare Zeit gesichert:

„Zielsetzung: Ein Hinüberwechseln der Länder Südostasiens in das kommunistische Lager zu verhindern und ihnen dabei zu helfen, den Willen und die Fähigkeit zu entwickeln, der inneren und äußeren kommunistischen Bedrohung Widerstand entgegenzusetzen und zur Stärkung der Freien Welt beizutragen." (1)

Washington hat mit dem Waffenstillstandsabkommen seine Vorstellungen von einem ,ehrenhaften“ Rückzug aus Vietnam voll durchsetzen können, während die vietnamesische Seite Schritt für Schritt vor den amerikanischen Forderungen zurückweichen mußte. Dies wird deutlich, wenn man sich die erstaunlich exakte Übereinstimmung des Waffenstillstandsabkommens mit den bereits im Januar 1969 veröffentlichten Vorstellungen Henry Kissingers zur Lösung des Vietnamproblems vor Augen fuihrt (2).

Schon damals hatte es Kissinger als Fehler bezeichnet, Saigon und die Provisorische Revolutionsregierung zu den bis Januar 1969 verzögerten Verhandlungen in Paris hinzuzuziehen: Einerseits müsse es zunächst darum gehen, mit der kommunistischen Seite Übereinstimmung über die umfassenderen Ziele und

+) Damit soll an den Aufsatz B. Rabehls in: Probleme des Klassenkampfs 6 angeknüpfit werden; allerdings weniger in der Absicht, sich von den dort vertretenen Ansichten abzusetzen, als vielmehr in dem Bemühen, die dort angerissenen Fragen zuzuspitzen und weiter zu klären.

1) Konzeption des Nationalen Sicherheitsrats zur Südostasienpolitik der USA 1952 , Dokument Nr. 2, The Pentagon Papers, New York (Bantam) 1971, S. 27.

2) Henry A. Kissinger, The Viet Nam Negotiations, in: Foreign Affairs, Januar 1969, S. 211 ff. - Ein halbes Jahr nach Erscheinen dieses Aufsatzes wurde Kissinger - der 1967 bereits schon einmal in einer geheimen Vietnam-Mission tätig geworden war - Nixons Sicherheitsberater. 
Vorstellungen für die Zukunft des südostasiatischen Raumes zu erzielen (3) andererseits muisse so lange wie möglich die Frage des Status der Provisorischen Revolutionsregierung offengelassen werden (4).

Genau dieser Strategie entsprachen die im August 1969 begonnenen streng vertraulichen Gespräche zwischen Kissinger und Le Duc Tho (5). Vertragsschließende waren im Januar 1973 die Regierungen in Washington und Hanoi; Saigon und die Revolutionsregierung haben sich dem Abkommen nur indirekt angeschlossen. Nirgends in dem Abkommen (6) wird der Status der südvietnamesischen Regierungen angesprochen, weshalb noch vor Unterzeichnung Thieu und Washington erklären konnten, daß auch weiterhin für sie das Saigoner Regime die einzige rechtmäßige Regierung Siidvietnams bleibt. - In Kissingers Worten:

„,Einer souveränen $\mathbb{R}$ egierung ist es freigestellt, mit jeder beliebigen Gruppe Gespräche zu führen, die eine relevante einheimische Machtbasis besitzt, ohne dieser dadurch eigene Souveränität zu verleihen; dasselbe passiert alle Nasen lang bei Verhandlungen mit Gewerkschaften oder sogar im Rahmen der Arbeit der Polizei." (7)

Alle Fragen eine südvietnamesische Koalitionsregierung oder üöerhaupt die politische Zukunft des Südens betreffend sollten von dem Übereinkommen zwischen Washington und Hanoi ausgeklammert und den Auseinandersetzungen zwischen den südvietnamesischen Parteien überlassen bleiben, weil diese am ehesten mit den für den Außenstehenden völlig undurchsichtigen lokalen Machtverhältnissen vertraut seien. Allenfalls könnte eine gemischte Kommission gebildet werden, die sich mit der Wiederherstellung einer einheitlichen politischen Gewalt mittels allgemeiner Wahlen befaßt.

Nichts anderes ist der Substanz nach in dem Abkommen von Paris vereinbart worden, aber auch ebenso vage sind die diesbezüglichen Vorkehrungen geblieben: Die vierseitige gemeinsame Militärkommission ist inzwischen - nach Abzug der letzten US-Soldaten - aufgelöst worden. Die nach Art. 17 des Abkommens gebildete gemeinsame Militärkommission hat sich Ende April zwar noch einmal über den Austausch von Zivilgefangenen einigen können; zur Realisierung des Waffenstillstands hat sie bisher jedoch keinen Beitrag geleistet (8). Der nach Art. 12 vorgesehene dreiseitige ,Nationale Rat der Nationalen Versöhnung und Eintracht"

3) Vgl. ebda. S. 229.

4) Vgl. ebda. S. $224 \mathrm{f}$.

5) Die Außenministerin der Revolutionsregierung bestätigte in einem Spiegel-Interview, daß ihre Vertreter mit keinem der bevollmächtigten amerikanischen Unterhändler in Kontakt gekommen war. Spiegel 22.5.1972, S. 90 f. - Die von Hanoi gegebene Begründung für den Ausschluß der Vertreter Saigons von den Geheimverhandlungen nämlich, daß ,die Thieu-Regierung nicht die legale Regierung Südvietnams sei“" - müßte analog auch für die Revolutionsregierung gelten. Vg1. Süddeutsche Zeitung, 16. 11. 1972.

6) Text in $S Z 27 . / 28.1 .1973, \mathrm{~S} .49 \mathrm{f}$.

7) K.ssinger, a. a. O. S. 232 .

Aufgrund des ungeklärten Status der NLF-Regierung hat es für die Vietkong-Vertreter von Anfang an keine realistischen Arbeitsmöglichkeiten gegeben. Die NLF-Vertreter werden wie Gefangene gehalten und sind häufig tätlichen Angriffen ausgesetzt. Vgl. etwa S2z 26.2.1973. 
ist bisher gar nicht zustandegekommen; die sehr spät begonnenen Verhandlungen zwischen Saigon und NLF in Paris, die bis Ende April zum Abschluß eines „Abkommens über die inneren Angelegenheiten Südvietnams ${ }^{66}$ und zur Einleitung allgemeiner Wahlen fuihren sollten, haben sich in der Gegenüberstellung der gegensätzlichen Standpunkte festgefahren (9).

Schließlich sprach sich Kissinger von Anfang an gegen ein formales Waffenstillstandsabkommen aus, das notwendigerweise das Territorium der gegnerischen Parteien klar abstecken und damit politische Entscheidungen vorwegnehmen würde; stattessen sollte man auf eine stillschweigende de facto Einstellung der Kampfhandlungen hinarbeiten (10).

Tatsächlich hat sich auch diese amerikanische Position durchgesetzt: Indem sich die Pariser Verhandlungspartner für unfähig erklärten, das von den beiden Parteien jeweils besetzte Gebiet wenigstens grob abzustecken, blieb der Waffenstillstand eine Farce. Jede Möglichkeit der Option der südvietnamesischen Bevölkerung für die eine oder andere Seite wurde ausgeschlossen, und die Herstellung freier politischer Betätigungsmöglichkeiten völlig dem Belieben des jeweiligen Machthabers unterworfen (11). Die Kämpfe um den territorialen Besitzstand ent brannten heftiger denn je. Das Waffenstillstandsabkommen wird von keiner Seite ernst genommen. Auch die Internationale Kontroll- und Uberwachungskommission ist völlig hilflos und wirkungslos gehalten worden, genau wie nach dem Genfer Abkommen von 1954 (12). Nachdem in letzter Zeit immer häufiger Hubschrauber der ICCS beschossen und zum Absturz gebracht wurden, drohen Kanada und Indonesien jetzt mit dem Auszug bis Ende Juni.

Die Übereinkunft von Paris erweist sich damit lediglich als Instrument des „ehrenvollen" Rückzugs der USA und der anderen Großmächte aus dem Krieg und zur Isolierung des Konflikts auf das Gebiet Südvietnams; der Befreiung von imperialistischer Unterdrückung und Ausbeutung ist das vietnamesische Volk damit keinen Schritt nähergekommen.

II

Wie konnte sich die kommunistische Seite mit einer derartig nichtssagenden Entlassung der USA aus der Verantwortung einverstanden erklären?

Noch im Mai 1969 hatte die Provisorische Revolutionsregierung in Paris in ihrer 10-Funkte-Erklärung eine Koppelung jeder Waffenstillstandsverainbarung mit bestimmten unverzichtbaren politischen Regelungen gefordert: Bis zum vollständigen Abzug der USA und zur endgültigen Entscheidung über die Regierungs-

9) Vgl. SZ 26. 4. 1973. Während Saigon auf der Diskussion des nordvietnamesischen Truppenabzugs als erstem Tagesordnungspunkt beharrt, halten die PRR-Vertreter die Herstellung demokratischer Freiheitsrechte in Sudvietnam für vorrangig.

10) Vgl. Kissinger, a. a. O. S. 226 f.

11) Seit Mai finden im Saigoner Machtbereich bereits Wahlen zu Gemeinde- und Dorfverwaltungsräten statt; wie bisher ist der Vietkong nicht zugelassen! Vgl. S2 9. 5. 1973.

12) Die mindeste Schlußfolgerung aus den Erfahrungen seit 1954 wäre die gewesen, der ICCS den technischen Apparat der UNO zur Verfügung zu stellen. Vgl. John S. Hannon Jir, The International Control Commission Experience and the Role of an Improved International Supervisory Body in the Vietnamese Settlement, in: Virginia Joumer International Law, vol. 9 (1968), S. 20 ff. 
bildung mittels allgemeiner Wahlen sollte eine Koalitionsregierung bestehend aus Vertretern aller politischen Gruppen die Regierungsgewalt wahrnehmen; die führenden Köpfe der Saigoner Marionettenregierung sollten davon jedoch ausgeschlossen sein (13). Das sollte sicherstellen, daß auf keinen Fall die Saigoner Marionetten die Hebel der Staatsmacht in der Hand behielten, um die Kommunisten zur Durchsetzung der vertraglichen Vereinbarungen auf den Weg der Gewaltanwendung verweisen zu können, wie es nach 1954 mit den vorgesehenen Wahlen geschehen war.

Die Verhandlungsposition des 10-Punkte Programms konnte sich auf die Erfolge der Tet-Offensive 1968 stützen, wo die vietnamesische Befreiungsbewegung bewiesen hatte, daß die übergroße Mehrheit der Bevölkerung Südvietnams aktiv hinter ihr stand; gleichzeitig hatte die überraschende Eroberung tausender südvietnamesischer Ortschaften, Provinzhauptstädte, und der ehemaligen Hauptstadt Hue die noch im Dezember 1967 von General Westmoreland in Washington genährten Hoffnungen auf einen amerikanischen militärischen Sieg zusammenbrechen lassen. Die Folge war die Einschränkung der Bombardierungen der DRV, der Verzicht Johnsons auf die Präsidentschaftskandidatur und die Einleitung der Verhandlungen in Paris.

Hier lag allerdings der einzige schwache Punkt der Konzeption Kissingers: Wie konnten die USA die in Südvietnam kämpfenden Parteien sich selbst überlassen, wenn sich das korrupte Saigoner Regime und dessen Streitkräfte als völlig unfähig erwiesen hatten, dem Ansturm der Befreiungsbewegung ohne amerikanische militärische Unterstützung zu widerstehen?

An diesem Punkt schien die amerikanische Position hoffnungslos, weil der Kern des Problems nicht materieller oder militärischer Natur war, sondern politischer. Daß sie dieses Defizit bei aller Anstrengung nicht ausgleichen konnte, schien die Johnson-Administration endlich eingesehen zu haben; dementsprechend signalisierte auch die vietnamesische Seite durch allmähliche Reduzierung der Angriffshandlungen und Rückzug einiger nordvietnamesischer Divisionen hinter die Entmilitarisierte Zone noch im Verlauf des Jahres 1968, daß sie zur allmählichen Herstellung eines de facto Waffenstillstands als Vorbedingung für eine politische Kompromißlösung bereit war (14). Die Johnson-Regierung zögerte ernsthafte Verhandlungen jedoch noch hinaus; und Nixon machte gleich nach seinem Amtsantritt deutlich, daß er sich zu keinem Kompromiß bereitfinden wollte, indem er die freigewordene Bomberkapazität nur umso gezielter gegen den Süden des Landes richten ließ (15).

Auch Kissinger sah die Situation 1968/69 nicht so negativ. Der Verlauf der

13) Punkt 5 der 10-Punkte-Erklärung; Text in: Richard A. Falk (Hrsg.), The Vietnam War and International Law, Bd. 3, Princeton 1972. S. $897 \mathrm{ff}$.

14) Zahlreiche Beobachter sprechen von einem stillschweigenden Übereinkommen zwischen NLF und USA. Vgl. die eingehende Analyse von J.L.S.Girling, Nixon's ,Algeria“"-Doctrine and Disengagement in Indochina, in: Pacific Affairs, 4/44 (Winter 1971-72) S. 527 ff.

15) Vgl. ebda. und das Interview mit dem von Nixon abgelösten Verhandlungsführer in Paris Harriman, in: New York Times Magazine 24. 8. 1969. 
Tet-Offensive hatte seiner Meinung nach vor allem bewiesen, daß die Kommunisten aufgrund ihrer materiellen Schwäche nicht in der Lage waren, ihre politischen Erfolge auf Dauer abzusichern. Darüberhinaus ließe die zunehmende Abhängigkeit von ausländischer materieller Unterstützung insbesondere aus China und der Sowjetunion den politischen Vorteil der Befreiungsbewegung zusammenschrumpfen. Und schließlich könnten die USA diesem Prozeß auch durch eine entschiedenere Strategie nachhelfen:

„Wir sollten eine Strategie verfolgen, die die Verluste reduziert und sich auf den Schutz der Bevölkerung konzentriert. Wir sollten mit der Stärkung der vietnamesischen Armee fortfahren, um den schrittweisen Rückzug eines Teils der amerikanischen Streitkräfte zu ermöglichen, und wir sollten Saigon drängen, seine Basis zu erweitern, um es für die politische Auseinandersetzung mit den Kommunisten zu stärken, die es früher oder später angehen muß." $(16)$

Dies ist die von Nixon eingeleitete Strategie der "Vietnamisierung“ des Krieges. Einerseits besteht sie in der erzwungenen „Urbanisierung" Südvietnams, wie sie von dem ebenfalls einflußreichen Vietnamberater S. P. Huntington bereits im Sommer 1968 als ,endlich gefundene Lösung des Problems der Befreiungskriege " propagiert wurde (17). Durch intensive Bombardierung des Landes wird die Bevölkerung in die Städte getrieben, wo sie in Flüchtlingslagern unter strengster Bewachung von jeglichem Kontakt mit der Befreiungsbewegung abgeschnitten wird (18). - Andererseits wird mit einem ungeheuren Aufwand an Material und finanziellen Anreizen die Armee und der eng mit ihr verknüpfte Verwaltungsapparat Saigons aufgemöbelt und an die selbständige Bedienung des Materialkrieges gegen den Vietkong gewöhnt.

Durch den brutalen Bombeneinsatz wurden die amerikanischen Bodentruppen allmählich überflüssig. Im Mai 1969 kündigte Nixon die erste Reduktion der damals 540000 Mann zählenden amerikanischen Truppen in Vietnam an. Parallel dazu verfügt er die Ausweitung des Krieges auf Kambodscha im Mai 1970 und - als die NLF immer noch an ihren Forderungen vom Mai 1969 festhält (19) den Einmarsch sïdvietnamesischer Truppen nach Laos und die massivsten Bombardierungen des „Ho Chi Minh-Pfades" in der Geschichte des Krieges. Obwohl die Laos-Operation für die Saigoner Armee ein Desaster wird, bleibt ein Versuch der Befreiungsbewegung aus, diese Schwäche der Imperialisten innerhalb Südvietnams auszunutzen. - Die Rechnung Washingtons geht auf.

In dem 7-Punkte-Plan der Provisorischen Regierung vom 1.7. 1971 wird der Waffenstillstand und die Herausgabe der amerikanischen Kriegsgefangenen nur noch von der Zusicherung eines Abzugstermins für die US-Truppen und von der Einstellung dieser Vietnamisierungsstrategie, d.h. der weiteren Stärkung des

16) Kissinger, op. cit. S. $233 \mathrm{f}$.

17) Vietnam: The Bases of Accomodation, in: Foreign Affairs, Juli 1968, S. 642 ff.

18) Vgl. Committee of Concerned Asian Scholars, The Indochina Story, New York (Bantam) 1970, insbes. Kap. 11. Dazu auch Raphael Littauer/Norman Uphotf (Hrsg.), The Air War in Indochina, Air War Study Group, Cornell University, Boston 1972.

19) 8-Punkte-Erklärung der PRReg. vom Sept. 1970, in: Falk, a. a. O. S. 900 ff. 
Thieu-Regimes abhängig gemacht; in Saigon soll eine neue Administration unter Beteiligung aller nichtkommunistischen Gruppen gebildet werden, die mit der Revolutionsregierung in Verhandlungen über die Schaffung einer dreiseitigen Koalitionsregierung bis zur Durchfuhrung allgemeiner Wahlen eintreten soll (20).

Mit der gleichzeitigen Ankunft Le Duc Thos in Paris und der Ablösung des amerikanischen Verhandlungsfürers durch den ehemaligen stellvertretenden Botschafter in Saigon, Porter, treten die Verhandlungen in Paris erstmals in ein ernsthaftes Stadium. Der internationale Kontext fur die Verhandlungen hat sich jedoch inzwischen ebenfalls entscheidend gewandelt: Am 18. Juli 1971 werden die Vorbereitungen für eine Reise Nixons nach Peking bekanntgegeben (21).

In den folgenden 18 Monaten wird Hanoi von Washington mit einem präzise terminierten Wechsel von vertraulicher Verhandlungsführung und sich steigernden Terrorangriffen unter Druck gesetzt. Als die Geheimgespräche zwischen Tho und Kissinger $a b$ Ende Juli 1971 nicht zu dem von den USA geforderten Ergebnis führen, steigert die US-Luftwaffe ihre, ,reaktiven Schutzangriffe" gegen die DRV bis Weihnachten zu seit 1968 nicht gekannter Stärke. Nach Beendigung dieser Bombardierungen kommt man am 27. Januar 1972 wieder in Paris zusammen, wo Porter einen bereits in den Geheimverhandlungen erörterten 8-Punkte-Plan der USA vorlegt, der bis auf 2 Steilen mit den Vorstellungen Hanois ubereinstimmt: im Grunde geht es nur noch um die Frage der weiteren amerikanischen Unterstützung des Thieu-Regimes. Weil die DRV/NLF an der Forderung nach Aufgabe Thieus festhält, kommt es Anfang Februar erneut zum Abbruch der Verhandlungen in Paris. Danach beginnt ein amerikanischer Luftwaffen- und Marineaufmarsch bis dahin ungekannten Ausmaßes; die auf Südvietnam und die DRV abgeworfene Bombenlast wird innerhalb weniger Tage verzehnfacht! - Der gleichzeitige herzliche Empfang, der Nixon in Peking bereitet wird (21.-28. Febr.), kann von Hanoi nicht anders denn als Einverständnis begriffen werden.

Die Früjahrsoffensive des Vietkong, die dann am 31 . März begann, muß als verzweifelter Versuch gelten, eine längst verlorene Position doch noch zuruickzugewinnen. Es zeigte sich deutlich, daß die neue amerikanische Strategie tatsächlich darin erfolgreich war, das politische Defizit wettzumachen: Unter dem furchtbarsten Bombenhagel der Geschichte wurde für die große Masse des vietnamesischen Volkes schließlich die Frage nach den politischen Herrschaftsverhältnissen irrelevant; anstatt den Guerilla ,wie das Wasser den Fisch ${ }^{66}$ zu umgeben, floh die Bevölkerung das Kampfgeschehen zu Zehntausenden, um nur das nackì Leben $\mathrm{zu}$

20) Text in: Internat. Herald Tribune, 2.7. 1971, S. 2.

21) Gleichzeitig wird bekannt, daß Peking die Einberufung einer neuen Indochinakonferenz befurwortet, was Hanoi bis dahin abgelehnt hatte. Vgl. SZ 16.7.1971. - Schon die Kambodscha-Invasion erfolgte in einer Atmosphäre demonstrativer Zurückhaltung der kommunistischen Großmächte: In geheimen Verhandungen bemühte sich die chinesische Regierung zwei Monate lang vergeblich um ein Arrangement mit dem Militärregime in Pnom Penh, um erst danach die Exilregierung Prinz Sihanouks anzuerkennen. Die SU weigert sich sogar bis heute, das Lon Nol-Regime zugunsten von Sihanouks Exilregierung fallenzulassen. Vgl. Girlins, a. a. O. S. 532, und zu Sihanouks Vorwürfen gegen die osteuropäischen Staaten SZ 24.4. 1973. 
retten (22). Die Hälfte der Bevölkerung Südvietnams ist von ihren Wohnsitzen auf dem Lande geflüchtet und drängt sich in den wenigstens vor Flächenbombardements und Artilleriefeuer sicheren Städten zusammen (23). Wenn die NLF auch $80 \%$ des Landes kontrolliert, so leben in diesem Gebiet doch nur noch $20 \%$ der Bevölkerung. Die Insassen der Flüchtlingslager und der Slums haben gar keine Möglichkeit mehr, aktiv am Kampf teilzunehmen (24). Anders als 1968 verhallte diesmal der Aufruf der NLF zur allgemeinen Erhebung ungehört (25).

Die Erfolge der Offensive erreichten auch bei weitem nicht melr das Ausmals vom Frïhjahr 1968. Nur dann hätte sich die Macht- und Verhandiungsposition der Vietnamesen gegenüber den USA verbessert, wenn die Befreiungsstreitkräfte größere Bevölkerungszentren hätten auf die Dauer halten können; genau das aber verhinderten die Bomben- und Napalmangriffe der amerikanischen Luftwaffe, die alles in Schutt und Asche legten, wo immer die Befreiungsarmee sich festsetzte (An Loc, Quang Tri).

Zwar mußten die USA - um das zu erreichen - noch einmal ihre Bodentruppen in den Kampf schicken und ihre See- und Luftstreitkräfte weiter auf einen neuen Rekordumfang verstärken, aber die Einschränkung ihres Truppenabbaus in Südvietnam oder etwa dessen Umkehrung konnten sie vermeiden (26). Darüberhinaus zeigten die massivsten Luftangriffe der Geschichte des Kieges gegen den Kern der nordvietnamesischen ökonomie, insbesondere Deichanlagen und Kraftwerke (Laser-Bomben), daß die Regierung in Washington vor keiner Brutalität mehr zurückschreckte, um Vietnam in die Knie zu zwingen. Als die Geheimgespräche zwischen Tho und Kissinger Anfang Mai jedoch immer noch keinen Sinneswandel bei den Nordvietnamesen erkennen lassen, verfigt IVixon die lange geplante, aber bisher nie gewagte Verminung der nordvietnamesischen Hä̂en $a b$ 9. Mai $1972(27)$.

Die Vorbereitungen für Nixons Reise nach Moskau Ende Mai laufen jedoch

22) Auf dem Höhepunkt der Kämpte berichtete die $S Z$ yom 4.5.1972: ${ }_{99}$ Allein im Raume Quang Tri und Hue sind über 200000 Flïchtlinge auf den Straßen, unter ihnen nach in Saigon vorliegenden Berichten bereits etwa ein Drittel der Bevölkerung Hues. Auch die Bevölkerung der Städte Kontum und Pleiku im Zentralen Hochland verläßt, soweit sie kann, die Städte."

23) Die Bevölkerungszahl Saigons beispielsweise hat sich vervierfacht. Lebten 1960 noch $85 \%$ der Bevölkerung auf dem Lande, so sind es jetzt nur noch $50 \%$ Vgl. Air War... \$. $62 \mathrm{ff}$.

24) Allein 1972 wurden durch die Kämpfe 2 Mio. neue Flüchtlinge geschaffen! Die Zahl der umgekommenen Zivilisten schätzt der Kennedy-Senatsausschuß fuir Flïchtlingsfragen auf 65000 . Vgl. SZ 6.12.1972. - In seinem Bericht vom Juli 1971 stellte derselbe Ausschuß für die Zeit seit 1965 bereits ca. 6 Mio. Flüchtlinge fest.

25) Vgl. SZ 26.1.1972. - Die Früjahrsoffensive 1972 scheint ganz überwiegend von regulären nordvietnamesischen Truppen geführt worden zu sein. Vg1. den Bericht der SZ vom 10.4. 1972, der sich allerdings auf amerikanische Militärs beruft.

26) Die US-Truppen stellten Ende Juli ihre aktiven Kampfeinsätze wieder völlig ein. - Im Mai erreichte ihre Zahl 100000 , bis 1 . September sank sie weiter auf unter 39000.

27) Gleichzeitig steigerten die Amerikaner den Bombenkrieg gegen die DRV auf bis zu 1100 Einsätze in 24 Stunden; in der Woche vom 20. bis 26. Juni gingen auf Indochina insgesamt 218700 Tonnen Bomben nieder. SZ 26.6.72. 
ungestört weiter, und auch aus Peking kommt nur papierener Protest. Hier wurde schlaglichtartig die Widersprïchlichkeit der sowjetischen ebenso wie der chinesischeıl Vietnampolitik erhellt: Der Waffen- und Militärhilfe der kommunistischen Staaten, ohne die Vietnam sich längst den barbarischen Angriffen der USA hätte beugen müssen, entsprach je länger der Krieg dauerte desto weniger das politisch-diplomatische Verhalten der Regierungen in Peking und Moskau.

So wenig diese Zusammenhänge hier abschließend beurteilt werden können, so sehr drängt sich doch der Eindruck auf, daß die sowjetische wie die chinesische Führung das Ziel der Befreiung der indochinesischen Völker von der Herrschaft des Imperialismus - aus welchen Gründen auch immer - ihren Interessen an guten Beziehungen $\mathrm{zu}$ den USA, günstigen Kreditbedingungen für Weizenkäufe und Industriegiiterimporte, Entspannung und Abrüstung in Europa, internationaler Anerkennung Chinas und Ausbau seiner Handelsbeziehungen zu kapitalistischen Ländern insbesondere Japan untergeordnet haben (28).

Nach einem weiteren Zwischenspiel begann schließlich Mitte August eine neue geheime Konsultationstätigkeit mit den Regierungen der Großmächte, die schließlich in die neue Gesprächsrunde Kissinger - Tho zwischen Ende September und Anfang Oktober einmündete; hier scheint sich Hanoi endlich dazu bereitgefunden zu haben, seine Forderung nach Aufgabe des Thieu-Regimes fallenzulassen.

Anfang November war Hanoi dann zum Abschluß eines Abkommens bereit, das lediglich die Modalitäten des amerikanischen Abzugs regelte, aber auf die Errichtung einer Interimsstaatsgewalt bis zur Schaffung einer gewählten Regierung verzichtet: D. h. das Saigoner Regime mit Thieu an der Spitze bleibt intakt, womit die politische Zukunft des Landes präjudiziert ist (29).

Offensichtlich brachte Thieu selbst dagegen noch so erhebliche Bedenken vor, daß Washington die Unterzeichnung noch einmal hinausschob. In den folgenden drei Monaten wurde Südvietnam zum letzten Mal mit den modernsten Waffen vollgestopft, und gleichzeitig wurden der Gegenseite nochı weitere Zugeständnisse abgerungen. Zwar lassen sich aus einem Vergleich der im Oktober veröffentlichten Vertragsbedingungen (30) mit dem endgültigen Vertragstext keine Gründe dafür finden, warum die USA zu Weihnachten 1972 noch einmal die furchtbarsten Flächenbombardements des Krieges auf Hanoi und andere Städte der DRV

28) Außenminister Rogers berichtete bezüglich möglicher Reaktionen der Großmächte auf die Verminung am 16.5. vor einem Kongreßausscnuß, daß Washington zu der Ansicht gekommen war, ,daß der UdSSR mehr an guten Beziehungen zu den USA gelegen sei als an einer Freihaltung der nordvietnamesischen Häfen von Minen." SZ 12. 9. 1972.

Vgl. dazu den Bericht von Victor Louis in den Londoner „Evening News" über das Drängen Podgornys in Hanoi auf ein Einlenken der vietnamesischen Regierung. SZ 19.6.1972. - Genauso sind auch nur die Zitate aus dem nordvietnamesischen Armee-Organ „Quan Doi“ zu verstehen, die sich gegen die ,perfiden diplomatischen Manöver" der USA richten, als zur gleichen Zeit Kissinger in Peking verhandelte! SZ 22. 6. 1972. - Ähnliche Andeutungen machte Kissinger laut SZ vom 26. 6. 1972.

29) Der Verbleib der nordvietnamesischen Truppen im Süden ist kaum als Zugeständnis zu werten; dieser Punkt der früheren Ausführungen Kissingers ist offensichtlich deshalb fallengelassen worden, weil der Abzug ohnehin kaum zu kontrollieren wäre.

30) Vg1. SZ 27. 10.1972. 
niedergehen ließen; die präventive Alarmbereitschaft Hanois (31) ab 5. 12. deutet aber darauf hin, daß es zumindest um weitere mündliche Zugeständnisse in den Verhandlungen ging. Auf jeden Fall muß die Zerstörung der letzten größeren Industrieanlagen der DRV die Möglichkeiten Hanois, etwa eine neue Offensive im Süden zu un terstützen, weiter reduziert haben, und allen Seiten wurde noch einmal in aller Brutalität deutlich gemacht, daß Washington auch in Zukunft jederzeit ungeachtet der „Weltmeinung“ bereit ist, zu allen verfügbaren Terrormaßnahmen zu greifen, falls die Entwicklung in Vietnam zu einer entscheidenden Machtverschiebung zu seinen Ungunsten führen sollte (32).

\section{III}

Der „ehrenvolle“ Rückzug der USA aus Südvietnam läßt ein Land zurück, dessen Verwüstungen es zu großen Teilen für die Reproduktion des menschlichen Lebens unbrauchbar machen (33), und ein Volk, das weitgehend aufgrund nackter Existenzbedrohung unfähig ist, an den Auseinandersetzungen um seine politische Zukunft noch aktiv teilzunehmen. Das Ringen um die gesellschaftlich-politischen Verhältnisse in Südvietnam ist dem Kampf zwischen der Saigoner Armee und den Befreiungsstreitkräften, bzw. nordvietnamesischen Truppen allein überlassen. Dieser Kampf findet aber da seine Grenze, wo das Thieu-Regime in seiner Existenz ernsthaft gefährdet ist.

Davon aber erscheint die Lage in Südvietnam inzwischen weit entfernt. Bereits nach dem Abkommen bleibt das Saigoner Marionettenregime in der Rolle der international anerkannten (34) und in den Städten und Ballungszentren vorherrschenden Regierung.

Das Regime hat es nicht nötig, seine terroristische Unterdrückung jeder oppositionellen politischen Tätigkeit in seinem Machtbereich zu lockern (35). So wie Diem nach 1954 arbeitete Thieu in den letzten Jahren systematisch an der Zerschlagung jeder möglichen Dritten Kraft in Südvietnam (36) und zog mit Hilfe

31) Vgl.SZ 6. 12.1972.

32) Vgl. die laufenden Drohungen führender amerikanischer Regierungsvertreter mit neuen Bombenangriffen; zuletzt Verteidungsminister Richardson, $\mathrm{SZ} 3.4 .1973$, u. SZ 24. 4. 1973.

33) Vgl, insbes. die Arbeiten der Biologen Westing und Pfeiffer in: „Science" und „Scientific American".

34) Die US-Regierung konnte jüngst eine Reihe von Staatsbesuchen des südvietnamesischen Militärdiktators bei verschiedenen westeuropäischen und südostasiatischen Regierungen durchsetzen. - Dagegen hielt es Washington nicht für nötig, eine Note bezüglich der angeblichen Waffenstillstandsverletzungen der DRV an die Signatarmächte des Pariser Abkommens auch der PRR zukommen zu lassen, ohne daß diesbezüglich von irgendeiner Seite ein Protest bekanntgeworden wäre. Vgl. SZ 26. 4. 1973.

35) Erst Mitte Mai wurden von einem Tag auf den anderen 25 politische Parteien und. Gruppierungen verboten; seitdem existiert legal nur noch die erst im Februar 1973 gegründete Regimepartei - der dritte derartige Versuch Thieus. Vgl. SZ 18. 5. 1973.

36) Für den Fall einer wider Erwarten doch noch eintretenden Schwäche des Thieu-Regimes stünde allenfalls der seit seiner Rückkehr 1968 nach Saigon ungemein vorsichtig taktierende General Minh zur Verfügung, der sowohl von den verschiedenen buddhistischen Gruppierungen als auch der NLF akzeptiert würde. 
amerikanischer Dollars eine Schicht vor. Kriegsgewinnlern an sich heran, für die das Bündnis mit den politischen Machthabern nicht Folge ist, sondern Voraussetzung für ökonomischen Einfluß und persönliche Bereicherung (37). Und auch in Zukunft werden die nötigen Bestechungsgelder reichlich fließen (38).

Unter diesen Umständen kann Saigon bereits für August dieses Jahres ,international kontrollierte allgemeine Wahlen "ins Auge fassen und für den Abzug der nordvietnamesischen Truppen eine entsprechende Reduzierung der eigenen über 1 Millionen Mann umfassenden Armee anbieten, wonach dann auch die „kriegsbedingten Beschränkungen" der demokratischen Freiheitsrechte aufgehoben werden können (39).

Die Revolutionsregierung dagegen ist weniger an baldigen Wahlen interessiert (40) als an der vorrangigen Durchsetzung freier politischer Betätigungsmöglichkeiten, die sich Saigon aber nur mit Waffengewalt abzwingen lassen würde. Auf einen entsprechenden neuen Versuch der gewaltsamen Machteroberung wird sich Nordvietnam jedoch in absehbarer Zeit kaum einlassen - noch viel weniger als nach 1954 , als es 6 Jahre lang zögerte, gegen Diems flagrante Verletzungen des Genfer Abkommens wieder zu den Waffen zu greifen. Vielmehr deutet alles darauf hin, daß Hanoi bis auf weiteres dem Wiederaufbau des Landes den Vorrang geben muß vor der Weiterverfolgung der Befreiung des Südens (41).

Die amerikanischen Behauptungen über verstärkte Aktivität nordvietnamesischęr Truppen in Laos und Kambodscha entbehren ebenfalls jeglicher Grundlage (42). Sie scheinen vielmehr dazu bestimmt, eine fadenscheinige Rechtfertigung abzugeben für den weiteren Einsatz amerikanischer Bomber gegen diese beiden Länder und die Einstellung der Entminung der nordvietnamesischen Häfen und der Wiederaufbauhilfeverhandlungen mit der DRV. Dahinter wird erneut Nixons Politik des Terrors und der Erpressung sichtbar, mit der er jetzt über Hanoi auch noch das

37) Zu den korrupten Praktiken Thieus vgl. vor allem Peter King, The Political Balance in Saigon, in: Pacific Affairs 3/44 (Herbst 1971) S. $401 \mathrm{ff}$.

38) Der US-Kongreß bewilligte Anfang Mai bereits Wiederaufbauhilfe für Südvietnam, Laos und Kambodscha in Höhe von 632 Mio. Dollar, wogegen die vertraglich zugesicherte Wiederaufbauhilfe für die DRV noch nicht einmal beantragt wurde.

39) Vgl. den in Paris vorgelegten Zehnpunkteplan Saigons nach SZ 26. 4. 1973. - In Saigon ist bereits wieder die Rede von einem neuen Anlauf zur Errichtung der berüchtigten Wehrdörfer.

40) Zunächst soll lediglich zu einer verfassunggebenden Versammlung gewählt werden. Vgl. SZ 17. 5. 1973 .

41) Vgl. SZ 17. 5. 1973, die sich auf amerikanische Geheimdienstberichte bezieht. - Schon der Rückzug der neu errichteten Raketenabschußrampen bei Khe San auf amerikanische Proteste hin deutete in diese Richtung. Die Behauptung der US-Regierung über neue massive Infiltrationen nordvietnamesischer Truppen und Waffen wurden inoffiziell von Washingtoner Regierungskreisen zurückgenommen und als ,Schüsse vor den Bug" bezeichnet; tatsächlich ist inzwischen von der Rückverlegung mindestens einer nordvietnamesischen Division hinter dic Demarkationslinje die Rede. (Vgl. SZ 2. 5. 1973). Selbst Saigon* glaubt nicht mehr an die immer wieder vorausgesagti neue Offensive des Vietkong. (Vgl. SZ 24.4.1973).

42) Vgl. die inoffiziellen Verlautbarungen aus amerikanischen Militärkreisen nach SZ 18. 4. 1973 u. 21. 4. 1973. 
Einlenken der Khmer Rouge in Kambodscha erzwingen will (43). Nach anfänglichem Sträuben ließ sich Hanoi Mitte Mai tatsächlich wieder auf Geheimverhandlungen zwischen Kissinger und Le Duc Tho ein.

\section{IV}

Diese Hinweise darauf, daß sich Moskau, Peking und Hanoi einstweilen mit der Herrschaft der USA in Südvietnam abgefunden haben, sind deutlich genug. Dennoch weiterhin auf einen entscheidenden Fortschritt des Kampfes und einen „endgiiltigen Sieg des vietnamesischen Volkes" ${ }^{6 \mathrm{zu}}$ hoffen, hieße auch, den amerikanischen Imperialismus gründlich zu unterschätzen; man kann sicher sein, daß sich Washington alle Garantien etwa gegen weitere sowjetische und chinesische Waffenlieferungen an die DRV hat geben lassen (44) und daß auch die vereinbarte Wiederaufbauhilfe nicht eher nach Hanoi fließen wird, als die USA sich vergewissert haben, daß sie nicht zur Unterstützung eines neuen Befreiungsversuchs Südvietnams verwendet wird.

Zweifellos blieb den Vietnamesen in der gegenwärtigen Lage gar keine andere Wahl, als dem vorliegenden Abkommen zuzustimmen. Dennoch drängt sich die Frage auf, warum der Krieg bis an einen Punkt fortgeführt wurde, wo das vietnamesische Volk wahrscheinlich auf Jahrzehnte hinaus seinen Unterdrückern hilflos ausgeliefert ist und für ein Abkommen, das bereits vier Jahre früher zu erhalten war.

Bei der bestehenden Quellenlage ist zur Beantwortung solcher und ähnlicher Fragen größte Vorsicht geboten. So viel kann aber auch heute bereits als sicher gelten: Die Charakterisierung des Vietnamkrieges als eines Volksbefreiungskrieges wurde in dem Maße problematisch, in dem die kommunistische Seite auf die von den USA 1964/65 begonnene Militarisierung des Krieges mit einer verzögerten, aber qualitativ entsprechenden Militarisierung einging; in dem die Hauptlast des Kampfes sich mehr und mehr von dem sich "wie der Fisch im Wasser" bewegenden Guerillaverband der NLF verlagerte auf die regulären Truppen der DRV, die immer schwereres Kriegsgerät einsetzten und auch immer stärker von den Lieferungen aus der Sowjetunion und China abhängig wurden.

Die Vorstellung vom Guerillero, dem mit allen konventionellen Waffen nicht beizukommen ist, erwies sich spätestens ab 1968 als zu kurz gegriffen. In dem Maße, wie die Bambusfalle sich gegenüber Splitterbomben, Napalm und Defoliation als unwirksam erwies und durch Artillerie, Panzer und Flugabwehrraketen ersetzt werden mußte, in demselben Maße ging auch der Befreiungskampf der vietnamesi-

43) Vgl. Prinz Sihanouks Warnung an die USA und die DRV kurz nach Wiederbeginn zweiseitiger Geheimverhandlungen in Paris, nicht über die Köpfe der Betroffenen hinweg über die Zukunft Kambodschas zu verhandeln. ,Wir sträuben uns gegen eine Konferenz zwischen fremden Staaten, die unser Land zum Gesprächsgegenstand hat." SZ 30. 4. 1973.

44) Sowjetische Waffenlieferungen an Hanoi waren u. a. Thema der Gespräche Kissingers Anfang Mai in Moskau. - Daß man sich in diesem Punkt leicht einigen konnte, darf vermutet werden, nachdem das ZK der KPdSU kurz vorher offiziell die imperialistische Aggression in Vietnam für beendet erklärte! Vgl. ebda. 
schen Bauern über in einen von militärischen und politischen Experten zentral geleiteten konventionellen Krieg. So wenig die zivile Bevölkerung bei den Artillerieduellen und Panzerschlachten der Jahre seit 1968 eine Rolle spielte, so sehr drängt sich die Frage auf, wieweit die jahrelange Fortsetzung eines solchen Krieges von der Bevölkerung Südvietnams noch nachvollzogen und mit ihren Bedürfnissen vermittelt werden konnte (45).

Solche Fragen, die sich aus dem Wandel des Charakters des Krieges ergeben, hätten längst eine Revision der These über den Vietnamkrieg verlangt, die bis heute der internationalen Vietnamsolidarität aller Schattierungen zugrundeliegt:

„Das vietnamesische Volk erteilt uns täglich eine unübersehbare Lektion an Opfermut, Ausdauer und revolutionärer Menschlichkeit im Kampfe gegen den weltweiten Vertreter der Unterdruickung und Repression. Es beweist uns ununterbrochen, wie der nationale Befreiungskampf eines Volkes - sei es noch so klein einen erfolgreichen Kampf auch gegen die größte imperialistische Weltmacht führen kann. ${ }^{\text {" }}(46)$

Diese Auffassung, die mit ihrem ungebrochenen Optimismus in den letzten Jahren mehr und mehr den Blick auf die tatsächlichen Geschehnisse in Vietnam verschleierte und demoralisierende Tllusionen über die Realität des Imperialismus zu verbreiten half (47), erwies sich als so zählebig, gerade weil sie als Begriff der traumatischen Vietnamerfahrung der Studentenbewegung eine der Haupttriebkräfte des Wiedererstehens einer neuen Linken in den westlichen Ländern gewesen ist.

Was bis heute in Gestalt der Parole vom "Sieg" des vietnamesischen Volkes durch die Reden und Schriften der linken Gruppen und Sekten geistert, ist tatsächlich ein Mythos des Guerilleros (48), der zwar einer verzweifelten Intelligenz ursprünglich das Gefühl der Ohnmacht und Hilflosigkeit nahm und sie in die Lage versetzte, sich gegen die als unmenschlich erkannten Verhältnisse zur Wehr zu setzen (49). Indem es aber unter den Bedingungen einer immer noch im

45) Um diese Problematik müssen sich auch die Auseinandersetzungen in der Führung der vietnamesischen Arbeiterpartei 1968-69 gedreht haben, als es um die Frage ging, ob nach der Tet-Offensive die Militarisierung des Kampfes weiter intensiviert oder zur Guerillataktik zurückgekehrt werden sollte. Vgl. Girling, a. a. O. S. 530.

46) Gaston Salvatore/Rudi Dutschke, Einleitung zu Che Guevara, Brief an das Exekutivsekretariat von Ospaal, Berlin (Oberbaumpresse) 1967, S. 3.

47) Bezeichnend dafür ist etwa Paul M. Sweezy und Harry Magdoffs Editorial, U. S. Defeat in Vietnam - The Importance of Calling a Defeat a Defeat, in: Monthly Review 10/24 (März 1973) S. $1 \mathrm{ff}$, die dort bereits grundlegende Zweifel an ihrer bis dahin vertretenen These eingestehen müssen.

48) Vgl. dazu Günter Maschke, Kritik des Guerillero - Zur Theorie des Volkskrieges, Frankfurt (S. Fischer) 1973.

49) „Indem es uns gelang, die latente und manifeste Irrationalität des Systems, den Terror in den Institutionen und die Brutalität der Polizei in der Auseinandersetzung zu entlarven, versuchten wir - ähnlich wie der Vietkong - die Verwundbarkeit des Systems nachzuweisen. Gelungene Nachweise... halfen mit, die autoritäre Struktur des bürgerlichen Charakters in uns tendenziell zu zerstören, Momente der Ich-Stärke, der Überzeugung, das System als Ganzes in Zukunft doch stürzen zu können." Rudi Dutschke, Vom Antisemitismus zum Antikommunismus, in: Bergmann/Dutschke/ Lefèvre/Rabehl, Rebellion der Studenten, Reinbek (Rowohlt) 1968, S. 75 f. 
wesentlichen unentwickelten Klassenbewegung der Arbeiter kaum gelang, über den nur symbolischen Protest hinaus den ,Klassenkampf im eigenen Lande" durch Strategien sozialistischer Praxis konkret zu füllen, mußte die "Spannung zwischen dem abstrakt-moralischen Protest und der Unmöglichkeit, durch diesen Protest den Krieg gegen das vietnamesische Volk durch die Amerikaner zu beenden" (50) schließlich zu ihrer einseitigen Aufhebung führen in der Vorstellung, daß die entscheidenden Schlachten gegen den Imperialismus fern in der „Dritten Welt“ geschlagen werden.

Dieses Denken, das u.a. die allgemein konstatierbare, bestürzende Verdrängung der Schwierigkeiten sozialistischer Politik erst möglich machte, hält das Eingeständnis einstweiliger Niederlagen im antiimperialistischen Kampf nicht aus. Erst die selbstkritische Auseinandersetzung darüber jedoch kann die Flucht in bequeme Auswege verstellen und die Kritik der von den kommunistischen Staaten verkörperten traditionellen Antworten auf diese Probleme erzwingen, die so lange insbesondere mit dem Hinweis auf den vermeintlich unversöhnlichen antiimperialistischen Stand dieser Staaten in Vietnam abgebogen werden konnte (51).

50) Ebda. S. 76.

51) Zur Funktion dieser vorgeblichen gegenseitigen Ausschließlichkeit der beiden Lager für das Bewubtsein der westeuropäisclien Linken vgl. Rossana Rossanda, Die sozialistischen Länder: Ein Dilemma der westeuropäischen Linken, in: Kursbuch 30. 